\title{
Clinical Use of the Nanohydroxyapatite/Polyamide Mesh Cage in Anterior Cervical Corpectomy and Fusion Surgery
}

\author{
Hui Xu, ${ }^{1,2}$ Xiaofeng Ren, ${ }^{1}$ Dawei Wang, ${ }^{1}$ Yongfei Zhao, ${ }^{2}$ Yan Wang, \\ Geng Cui, ${ }^{2}$ Songhua Xiao, ${ }^{2}$ and Xuesong Zhang ${ }^{2}$ \\ ${ }^{1}$ Department of Orthopedics, Liaocheng People's Hospital, 67 Dongchang West Road, Liaocheng, Shandong Province 252004, China \\ ${ }^{2}$ Department of Orthopedics, Chinese People's Liberation Army General Hospital, Beijing 100853, China
}

Correspondence should be addressed to Yongfei Zhao; lakezyf@hotmail.com and Xuesong Zhang; zhangxuesong301@sina.com Received 13 October 2014; Accepted 26 December 2014

Academic Editor: Shuming Zhang

Copyright (C) 2015 Hui Xu et al. This is an open access article distributed under the Creative Commons Attribution License, which permits unrestricted use, distribution, and reproduction in any medium, provided the original work is properly cited.

Purpose. This study was to report the clinical use of biomimetic nanohydroxyapatite/polyamide 66 (n-HA/PA 66) mesh cages in anterior cervical corpectomy and fusion (ACCF) surgery. Method. 95 patients who underwent single level anterior cervical corpectomy and fusion for cervical spondylosis myelopathy (CSM) in our hospital were reviewed and divided into 2 groups according to using nanohydroxyapatite/polyamide mesh cage and titanium mesh cage (TMC). Demographic data of patients and surgical, clinical, and radiological data before operation and at last follow-up were collected and compared. Result. The operation time, surgical blood loss, complications, and Japanese Orthopaedic Association scores (JOA scores) of two groups were similar. At the last follow-up both the two groups obtained $100 \%$ solid bone fusion, but the TMC group had higher rate of severe cage subsidence than the n-HA/PA 66 group (27\% versus 2\%). Conclusion. Nanohydroxyapatite/polyamide 66 mesh cage is safe and effective in ACCF and can be a substitution to titanium mesh cage.

\section{Introduction}

Nanohydroxyapatite/polyamide 66 (n-HA/PA 66) is a biocomposite made by nanotechnology using hydroxyapatite and polyamide [1]. Nanotechnique is popular in biomaterial field and is widely accepted by researchers [2-4]. Hydroxyapatite (HA) and natural bone mineral have similar composition and structure, so HA was considered as an ideal bone repair material due to its osteoconductivity. However the brittleness of HA decide that it only can be used in no load-bearing bone repair. When the HA was used as a strut it easily broke $[5,6]$. Because chemical structure and active groups of polyamide (PA) are similar to collagen protein, it has good biocompatibility. PA also has excellent mechanical properties because of the strong hydrogen bonds in PA macromolecules $[7,8]$. N-HA/PA 66 composite combined the advances of the two materials. So it was considered as an ideal material in bone defect repair and reconstruction.
N-HA/PA 66 composite was developed by thermal-press molding technique [9]. The porosity of the materials is $36 \%$ to $57 \%$ and average diameter of the pores is $280 \mu \mathrm{m}$ to $500 \mu \mathrm{m}$. Good interface is provided by the irregular pores between n-HA/PA 66 and host bone; thus new bone tissue can grow in the pores. The porous n-HA/PA 66 composite is credited to have biological safety and to be effective $[10,11]$. Devices made by this material have been used for bone repair and reconstruction successfully in recent years [12-14].

N-HA/PA 66 composite cages for anterior cervical discectomy and fusion (ACDF) or anterior cervical corpectomy and fusion (ACCF) were reported by several researchers and show good clinical results [15-17]. They concluded that the n-HA/PA 66 composite cages were even better than the titanium mesh cages which are widely used in the world currently. However a new material needs more studies to verify the efficacy and safety before it is widely accepted and used, so we reviewed the patients who received the operation 


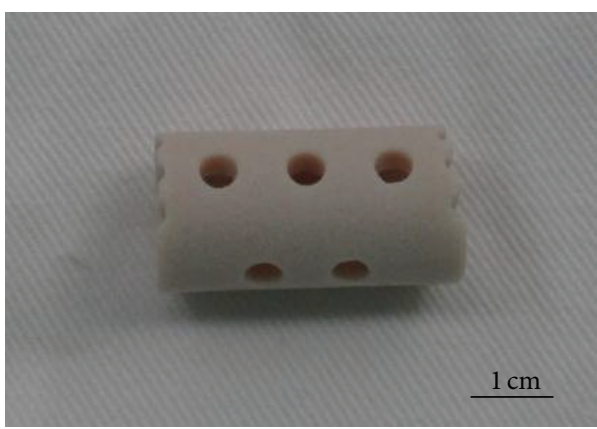

(a)

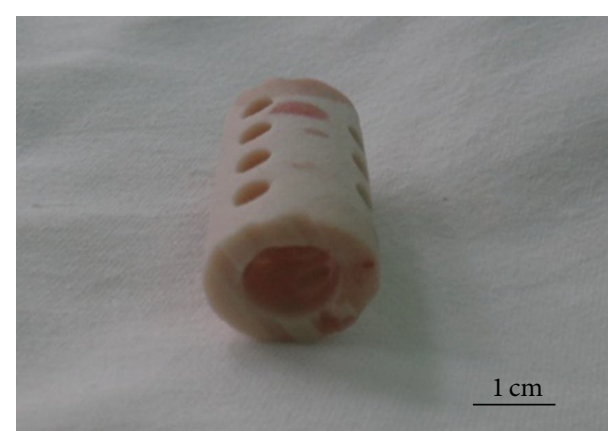

(b)

Figure 1: Lateral and axial view of the n-HA/PA 66 mesh cage.

of ACCF with nanohydroxyapatite/polyamide mesh cage and compared the outcomes to titanium mesh cage.

\section{Patients and Methods}

2.1. Patients. From January 2009 to June 2011, patients who underwent ACCF for cervical spondylosis myelopathy (CSM) in our hospital were reviewed. The patients' age, sex, height, photographs, radiographs, and clinical records were prospectively collected in our department database. The inclusion criteria were (1) patients with myelopathy, (2) the follow-up being at least 2 years, and (3) single-level ACCF surgery. The exclusion criteria were (1) cervical spondylosis radiculopathy or fracture, (2) cervical deformities or neoplasia, and (3) patients with multilevel corpectomy. 95 patients were included in this study with the age range $45-73$ years. The patients were divided into two groups by using n-HA/PA 66 composite cages or titanium mesh cages (TMC).

\subsection{N-HA/PA 66 Composite Cage. N-HA/PA 66 mesh cage} (Figure 1) (National Nano Technology Co. Sichuan, China) was made of n-HA/PA 66 composite and it has a series of length and diameter size for individual's variation. This product was approved by Chinese FDA in 2005. Figure 2 is the TEM micrographs of n-HA/PA 66.

2.3. Surgical Technique. After general anesthesia, the neck of patients was padded in extension position. The SmithRobinson method was used to perform anterior exposure [18]. Then the target cervical excision was confirmed by mobile digital imaging system, and cervical corpectomy and decompression were performed. The vertebral bone fragments were collected and used as graft material. The endplate of the upper and lower vertebrae was prepared with curette. Then TMC or n-HA/PA 66 mesh cages were selected with appropriate lengths, filled with local autologous bone, and implanted into corpectomy space (Figure 3). The anterior cervical plate was fixed to upper and lower cervical vertebrae with screws for further stabilization. After surgery, a soft cervical collar was used for six weeks.

2.4. Outcomes Assessment. Blood loss, operation time, complications of surgery, and hospital stay were recorded and

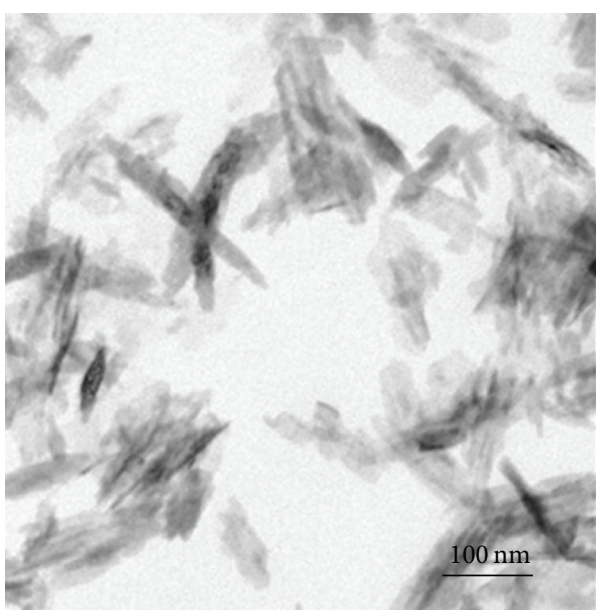

Figure 2: TEM micrographs of n-HA/PA 66.

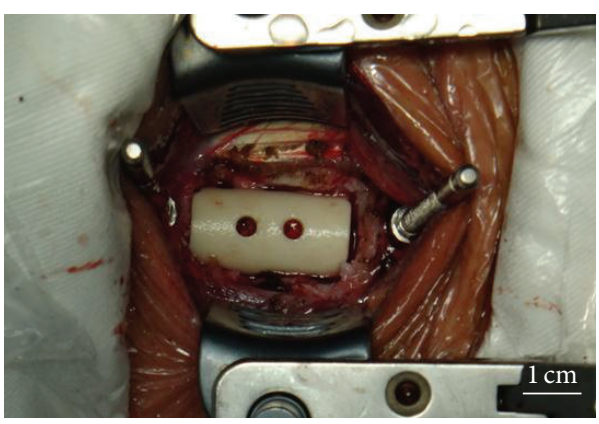

FIGURE 3: Implanting the cage after corpectomy.

compared between two groups. The Japanese Orthopedic Association (JOA) CSM scale was used to assess clinical result of each patient before surgery and at last follow-up.

The patients had radiological follow-up immediately, at one, three, and six months and then annually after surgery. The cervical spine radiographs were performed at each follow-up examination. Lateral plain radiographs were used to evaluate fusion status and cage subsidence. When trabecular bone grew across the interfaces, fusion was considered. If there was lucency between implants and 


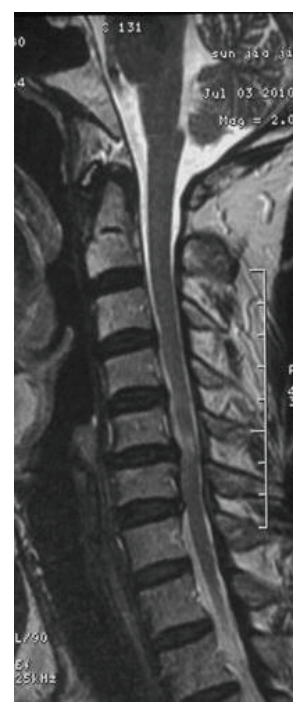

(a)

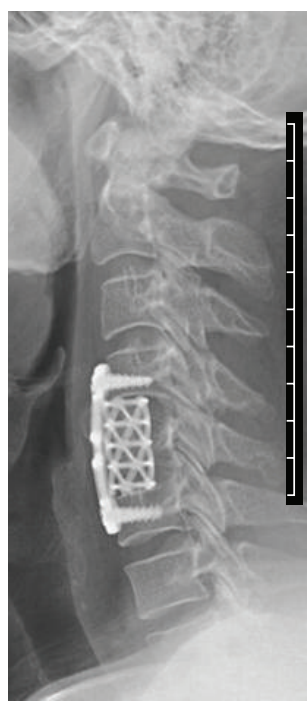

(b)

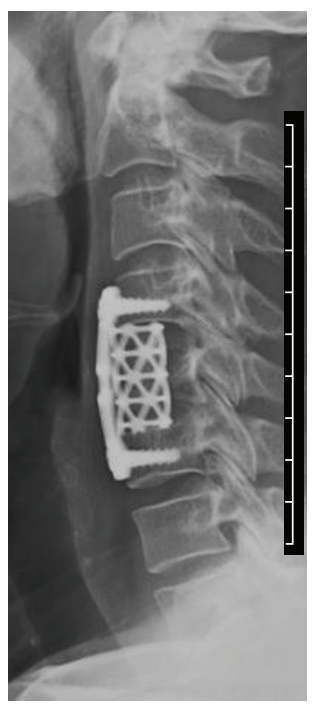

(c)

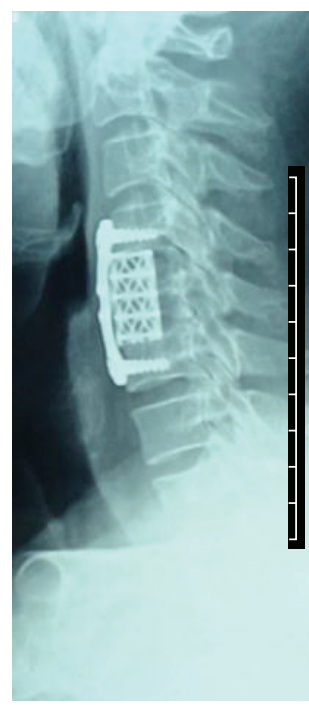

(d)

FIgURE 4: A 60-year-old man underwent anterior cervical corpectomy and fusion with a titanium mesh cage for CSM. (a) The compression located in the C5 level on the cervical MRI. (b) Lateral radiograph immediately after surgery. (c) 3 months after surgery the endplates were clear. (d) Two years after surgery the endplates were undefined and mild subsidence was observed.

vertebral endplates, nonunion would be considered. The change of fusion segments' height was defined as subsidence. When cage subsidence was bigger than three millimeters it was considered as severe subsidence. Five independent spine surgeons measured these radiographic parameters and the average value was calculated for final analysis.

2.5. Statistical Analysis. In this study all statistical analyses were performed using SPSS 17.0 statistic software (SPSS, Chicago, Illinois, USA). Student's $t$-test was used to compare mean data, and Pearson's Chi-squared test was performed for categorical data. $P<0.05$ was considered as statistical significance.

\section{Results}

The anterior fusion was performed using TMC mesh cage in 59 patients (Figure 4) and n-HA/PA 66 mesh cage in 36 patients (Figures 5 and 6). The demographics of cases are shown in Table 1. There was no statistical difference between these two groups (all $P>0.05$ ).

3.1. Surgical and Clinical Outcome. The operations were successfully performed in all patients. One patient of TMC group was reoperated 3 months after primary surgery because of screw loosening and plate displacement. No major procedure-related complications were found. There were no postoperative hematoma or wound infection in two groups. Some patients had dysphagia postoperatively, but they all were covered within 1 month. Duration of hospitalization, operation time, and blood loss were not statistically different between groups. The last follow-up JOA scores of both groups improved significantly compared to preoperative JOA scores.
TABLE 1: Demographic data of patients.

\begin{tabular}{lccc}
\hline & $\begin{array}{c}\text { n-HA/PA 66 } \\
(n=36)\end{array}$ & $\begin{array}{c}\text { TMC } \\
(n=59)\end{array}$ & $P$ \\
\hline M/F & $19: 17$ & $28: 31$ & 0.61 \\
Age (years) & $56.4 \pm 12.8$ & $58.6 \pm 14.3$ & 0.45 \\
Smoker/nonsmoker & $7: 29$ & $11: 48$ & 0.92 \\
Follow-up (months) & $30.6 \pm 10.8$ & $32.1 \pm 12.8$ & 0.56 \\
Pathogenic level & & & \\
C4 & 3 & 4 & \\
C5 & 10 & 19 & \\
C6 & 15 & 25 & \\
C7 & 8 & 11 & \\
\hline
\end{tabular}

Sex, age, smoker, and follow-up were not statistically different between two groups.

However, there was no statistical difference between groups (Table 2).

3.2. Radiographic Result. There was no case of cage breakage in the two groups at the last follow-up. However, there were 3 cases of screw loosening in TMC group. At the last follow-up, the fusion rate was $100 \%$ in two groups. 1 and 16 severe cage subsidence instances were observed in n-HA/PA 66 group and TMC group respectively, which have statistical difference (Table 3).

\section{Discussion}

Anterior cervical corpectomy and fusion (ACCF) is used when the anterior compression surpasses the disc level such 


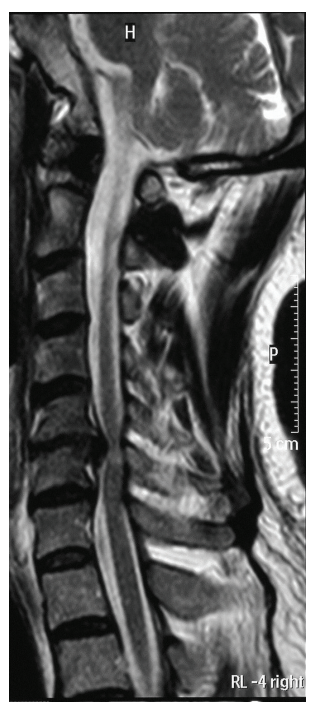

(a)

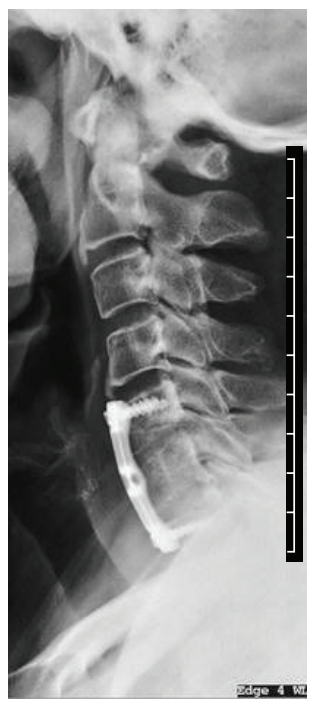

(b)

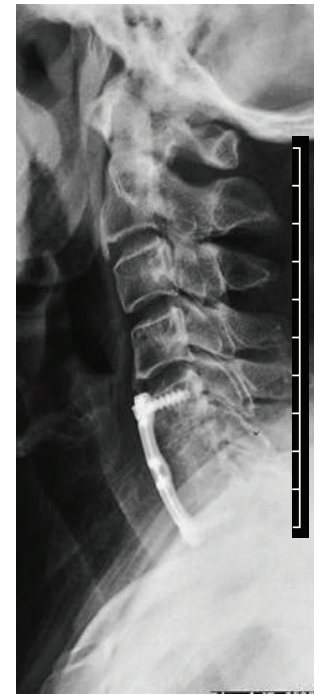

(c)

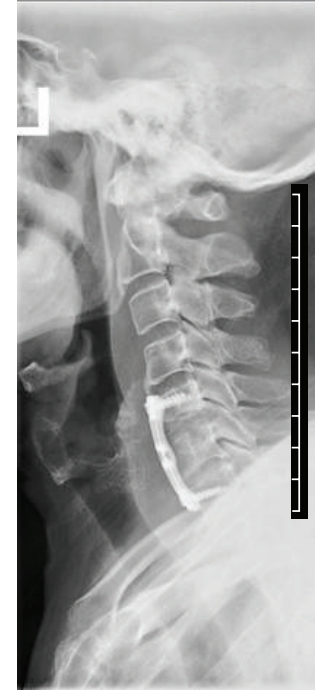

(d)

Figure 5: A 67-year-old man underwent cervical corpectomy and fusion with an n-HA/PA 66 mesh cage for CSM. (a) The compression located in the C6 level on the preoperative cervical MRI. (b) Lateral radiograph immediately after surgery. (c) 3 months after surgery. (d) Two years after surgery. The fusion status cannot be identified on the lateral radiograph, but cervical alignment, plate, and screws were not changed during follow-up.

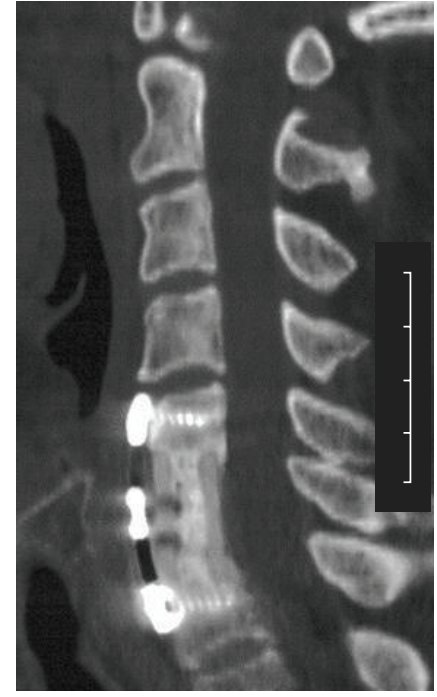

(a)

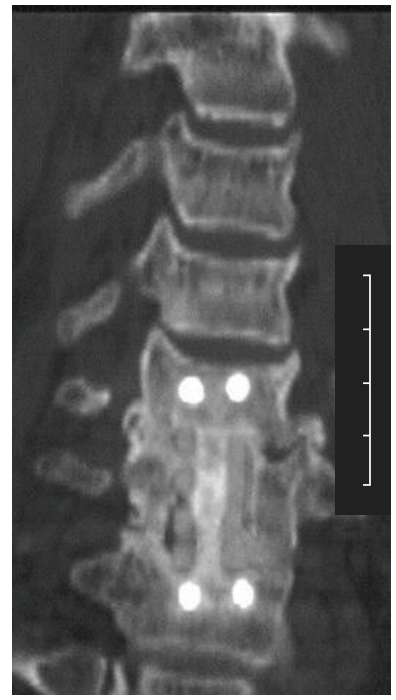

(b)

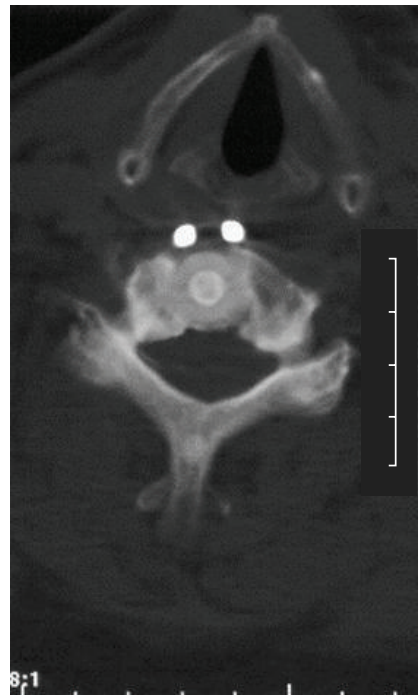

(c)

FIGURE 6: The same patient of Figure 5. One year after surgery solid fusion was seen on multiplane CT scan.

as ossification of the posterior longitudinal ligament (OPLL). Currently structural autografts, allografts, and cages are used as support structure implant in ACCF. However the donor site morbidity and the risk of disease transmission with allograft are concerns of many surgeons. Furthermore, there is much autologous local bone which can be used in the cage [19]. So cage devices become the best choice for cervical reconstruction. The cage filled with local bone can provide support, osteoinduction, and osteoconduction and has a high rate of osseous fusion.
Titanium mesh cage is widely used in anterior cervical corpectomy and fusion currently. And the clinical outcomes were favorable $[20,21]$. However the high rate of cage subsidence has been observed in the past researches. Chen et al. found that TMC subsidence (more than one millimeter) occurred in $79.7 \%$ of patients and severe subsidence (more than three millimeters) in $19 \%$ of cases. They also concluded that severe TMC subsidence was correlated with bad clinical outcomes [22]. More recently, polymethylmethacrylate (PMMA), polyetheretherketone (PEEK), and carbon fiber 
TABLE 2: Surgical and clinical outcomes.

\begin{tabular}{lccc}
\hline & $\begin{array}{c}\mathrm{n}-\mathrm{HA} / \mathrm{PA} 66 \\
(n=36)\end{array}$ & $\begin{array}{c}\text { TMC } \\
(n=59)\end{array}$ & $P$ \\
\hline Hospitalization (day) & $5.1 \pm 1.6$ & $4.9 \pm 1.8$ & 0.59 \\
Operation time (mins) & $120 \pm 24$ & $114 \pm 32$ & 0.33 \\
Blood loss (mL) & $124 \pm 56$ & $116 \pm 54$ & 0.49 \\
Dysphagia & 4 & 7 & 0.91 \\
JOA scale preop & $8.2 \pm 1.4$ & $8.5 \pm 1.8$ & 0.40 \\
Last follow-up & $14.1 \pm 1.6^{*}$ & $13.4 \pm 1.9^{*}$ & 0.07 \\
\hline
\end{tabular}

${ }^{*}$ Compared to JOA scale preop $P<0.05$.

TABLE 3: Radiographic result.

\begin{tabular}{lccc}
\hline & $\begin{array}{c}\text { n-HA/PA66 } \\
(n=36)\end{array}$ & $\begin{array}{c}\text { TMC } \\
(n=59)\end{array}$ & $P$ \\
\hline Cage breakage & 0 & 0 & 1 \\
Screw loosening & 0 & 3 & 0.28 \\
Solid fusion & 36 & 59 & 1 \\
Reoperation & 0 & 1 & 1 \\
Cage subsidence $^{*}$ & 1 & 16 & 0.006 \\
\hline
\end{tabular}

${ }^{*}$ Cage subsidence was statistically different between two groups.

reinforced polymer (CFRP) cages have been developed for ACCF. But they are not widely used currently. At present, many nanomaterials have been used for bone regeneration because of their satisfactory osteogenic activity [23, 24]. A new n-HA/PA 66/glass fibre composite was developed by Qiao et al. and is still in experiment stage $[25,26]$.

The clinical results of n-HA/PA 66 cage have been gradually reported in recent years. Zhao et al. reported a 94.3\% fusion rate and a $2.9 \%$ cage subsidence rate in their 35 patients [15]. Yang et al. found a fusion rate of $97 \%$ in 35 patients who underwent ACCF with an n-HA/PA 66 mesh cage fusion. They also compared the outcomes between the nHA/PA 66 mesh cage and the TMC [17]. In their single-level cervical corpectomy series, solid bony fusion was found in $84 \%$ of patients in the TMC group at one year and in $94 \%$ four years after surgery. Solid fusion was found in $94 \%$ of patients in the n-HA/PA 66 mesh group at one year and in $97 \%$ four years after surgery. The rate of severe cage subsidence was $22 \%$ in TMC group and 6\% in n-HA/PA 66 group. They also reported that the postoperative VAS and JOA points in TMC group were poorer than the ones in n-HA/PA 66 group. In our series, we found that the clinical results were similar between two groups. The operation time, surgical blood loss, complications, and JOA score of two groups were similar. At the last follow-up both the two groups obtained $100 \%$ solid fusion. But the TMC group had higher rate of severe cage subsidence than the n-HA/PA 66 group (27\% versus $2 \%$ ). We think the reason is that n-HA/PA 66 material has superior elasticity, good interface, and big contact surface which lead to sooner fusion. Severe cage subsidence can lead to screws loosing and plate displacement. One patient of TMC group underwent reoperation because of the symptoms caused by plate displacement in our cases.
There were several disadvantages in this research. First, this study was a retrospective research and further prospective study is necessary. Second, the method of estimating fusion rate was not accurate. The fusion in this study may be considered as "clinical fusion." Flexion and extension X-ray films, CT scan, and even MRI should be used to judge the fusion status in future researches.

In conclusion, the n-HA/PA 66 mesh cage had safety and efficiency in ACCF. It was successfully used in ACCF and had good outcomes that can be a substitution to traditional TMC cage.

\section{Conflict of Interests}

The authors have no conflict of interests.

\section{Authors' Contribution}

Xuesong Zhang and Yongfei Zhao contributed equally to this paper.

\section{References}

[1] M. Huang, J. Feng, J. Wang, X. Zhang, Y. Li, and Y. Yan, "Synthesis and characterization of nano-HA/PA66 composites," Journal of Materials Science: Materials in Medicine, vol. 14, no. 7, pp. 655-660, 2003.

[2] X. H. Liu, X. M. Li, Y. B. Fan et al., "Repairing goat tibia segmental bone defect using scaffold cultured with mesenchymal stem cells," Journal of Biomedical Materials Research Part B: Applied Biomaterials, vol. 94B, no. 1, pp. 44-52, 2010.

[3] X. M. Li, Y. Huang, L. S. Zheng et al., "Effect of substrate stiffness on the functions of rat bone marrow and adipose tissue derived mesenchymal stem cells in vitro," Journal of Biomedical Materials Research Part A, vol. 102, no. 4, pp. 1092-1101, 2014.

[4] X. Li, L. Wang, Y. Fan, Q. Feng, F. Z. Cui, and F. Watari, "Nanostructured scaffolds for bone tissue engineering," Journal of Biomedical Materials Research-Part A, vol. 101, no. 8, pp. 2424-2435, 2013.

[5] H. H. K. Xu and C. G. Simon Jr., "Fast setting calcium phosphate-chitosan scaffold: mechanical properties and biocompatibility," Biomaterials, vol. 26, no. 12, pp. 1337-1348, 2005.

[6] S. S. Kim, M. Sun Park, O. Jeon, C. Yong Choi, and B. S. Kim, "Poly(lactide-co-glycolide)/hydroxyapatite composite scaffolds for bone tissue engineering," Biomaterials, vol. 27, no. 8, pp. 1399-1409, 2006.

[7] S. I. Lee and B. C. Chun, "Mechanical properties and fracture morphologies of poly(phenylene sulfide)/nylon66 blendseffect of nylon66 content and testing temperature," Journal of Materials Science, vol. 35, pp. 1187-1193, 2000.

[8] Y. W. Wang, Q. Wu, J. Chen, and G. Q. Chen, "Evaluation of three-dimensional scaffolds made of blends of hydroxyapatite and poly(3-hydroxybutyrate-co-3-hydroxyhexanoate) for bone reconstruction," Biomaterials, vol. 26, no. 8, pp. 899-904, 2005.

[9] H. Li, Y. Li, Y. Yan, J. Li, A. Yang, and H. Xiang, "Fabrication of porous n-HA/PA66 composite for bone repair," Key Engineering Materials, vol. 330-332, pp. 321-324, 2007. 
[10] H. Wang, Y. Li, Y. Zuo, J. Li, S. Ma, and L. Cheng, "Biocompatibility and osteogenesis of biomimetic nanohydroxyapatite/polyamide composite scaffolds for bone tissue engineering," Biomaterials, vol. 28, no. 22, pp. 3338-3348, 2007.

[11] Y. Xiong, C. Ren, B. Zhang et al., "Analyzing the behavior of a porous nano-hydroxyapatite/polyamide 66 (n-HA/PA66) composite for healing of bone defects," International Journal of Nanomedicine, vol. 9, no. 1, pp. 485-494, 2014.

[12] X. Yang, Y. Song, L. Liu, H. Liu, J. Zeng, and F. Pei, "Anterior reconstruction with nano-hydroxyapatite/polyamide-66 cage after thoracic and lumbar corpectomy," Orthopedics, vol. 35, no. 1, pp. e66-e73, 2012.

[13] X. Yang, Y. Song, Q. Kong, Q. Gong, F. Pei, and C. Tu, "Short-term effectiveness of nano-hydroxyapatite/polyamide66 intervertebral cage for lumbar interbody fusion in patients with lower lumbar degenerative diseases," Zhongguo Xiu Fu Chong Jian Wai Ke Za Zhi, vol. 26, no. 12, pp. 1425-1429, 2012.

[14] Y. Liu, S. Liu, and X. Su, "Core decompression and implantation of bone marrow mononuclear cells with porous hydroxylapatite composite filler for the treatment of osteonecrosis of the femoral head," Archives of Orthopaedic and Trauma Surgery, vol. 133, no. 1, pp. 125-133, 2013.

[15] Z. Zhao, D. Jiang, Y. Ou, K. Tang, X. Luo, and Z. Quan, "A hollow cylindrical nano-hydroxyapatite/polyamide composite strut for cervical reconstruction after cervical corpectomy," Journal of Clinical Neuroscience, vol. 19, no. 4, pp. 536-540, 2012.

[16] X. Yang, L. Liu, Y. Song, Q. Kong, J. Zeng, and C. Tu, “Outcome of single level anterior cervical discectomy and fusion using nano-hydroxyapatite/polyamide-66 cage," Indian Journal of Orthopaedics, vol. 48, no. 2, pp. 152-157, 2014.

[17] X. Yang, Q. Chen, L. Liu et al., "Comparison of anterior cervical fusion by titanium mesh cage versus nanohydroxyapatite/polyamide cage following single-level corpectomy," International Orthopaedics, vol. 37, no. 12, pp. 2421-2427, 2013.

[18] Y. Fengbin, W. Xinwei, Y. Haisong, C. Yu, L. Xiaowei, and C. Deyu, "Dysphagia after anterior cervical discectomy and fusion: a prospective study comparing two anterior surgical approaches," European Spine Journal, vol. 22, no. 5, pp. 1147-1151, 2013.

[19] A. M. T. Chau, L. L. Xu, J. H.-Y. Wong, and R. J. Mobbs, "Current status of bone graft options for anterior interbody fusion of the cervical and lumbar spine," Neurosurgical Review, vol. 37, no. 1, pp. 23-37, 2014.

[20] K. R. Eck, K. H. Bridwell, F. F. Ungacta, M. A. Lapp, L. G. Lenke, and K. Daniel Riew, "Analysis of titanium mesh cages in adults with minimum two-year follow-up," Spine, vol. 25, no. 18, pp. 2407-2415, 2000.

[21] A. E. Castellvi, A. Castellvi, and D. H. Clabeaux, "Corpectomy with titanium cage reconstruction in the cervical spine," Journal of Clinical Neuroscience, vol. 19, no. 4, pp. 517-521, 2012.

[22] Y. Chen, D. Chen, Y. Guo et al., "Subsidence of titanium mesh cage: a study based on 300 cases," Journal of Spinal Disorders and Techniques, vol. 21, no. 7, pp. 489-492, 2008.

[23] X. M. Li, H. F. Liu, X. F. Niu et al., "Osteogenic differentiation of human adipose-derived stem cells induced by osteoinductive calcium phosphate ceramics," Journal of Biomedical Materials Research-Part B Applied Biomaterials, vol. 97, no. 1, pp. 10-19, 2011.

[24] X. M. Li, H. F. Liu, X. F. Niu et al., "The use of carbon nanotubes to induce osteogenic differentiation of human adipose-derived
MSCs in vitro and ectopic bone formation in vivo," Biomaterials, vol. 33, no. 19, pp. 4818-4827, 2012.

[25] B. Qiao, J. Li, Q. Zhu et al., "Bone plate composed of a ternary nano-hydroxyapatite/polyamide 66/glass fiber composite: biomechanical properties and biocompatibility," International Journal of Nanomedicine, vol. 9, no. 1, pp. 1423-1432, 2014.

[26] B. Su, X. Peng, D. Jiang et al., "In vitro and in vivo evaluations of nano-hydroxyapatite/polyamide 66/glass fibre (nHA/PA66/GF) as a novel bioactive bone screw," PLOS ONE, vol. 8, no. 7, Article ID e68342, 2013. 

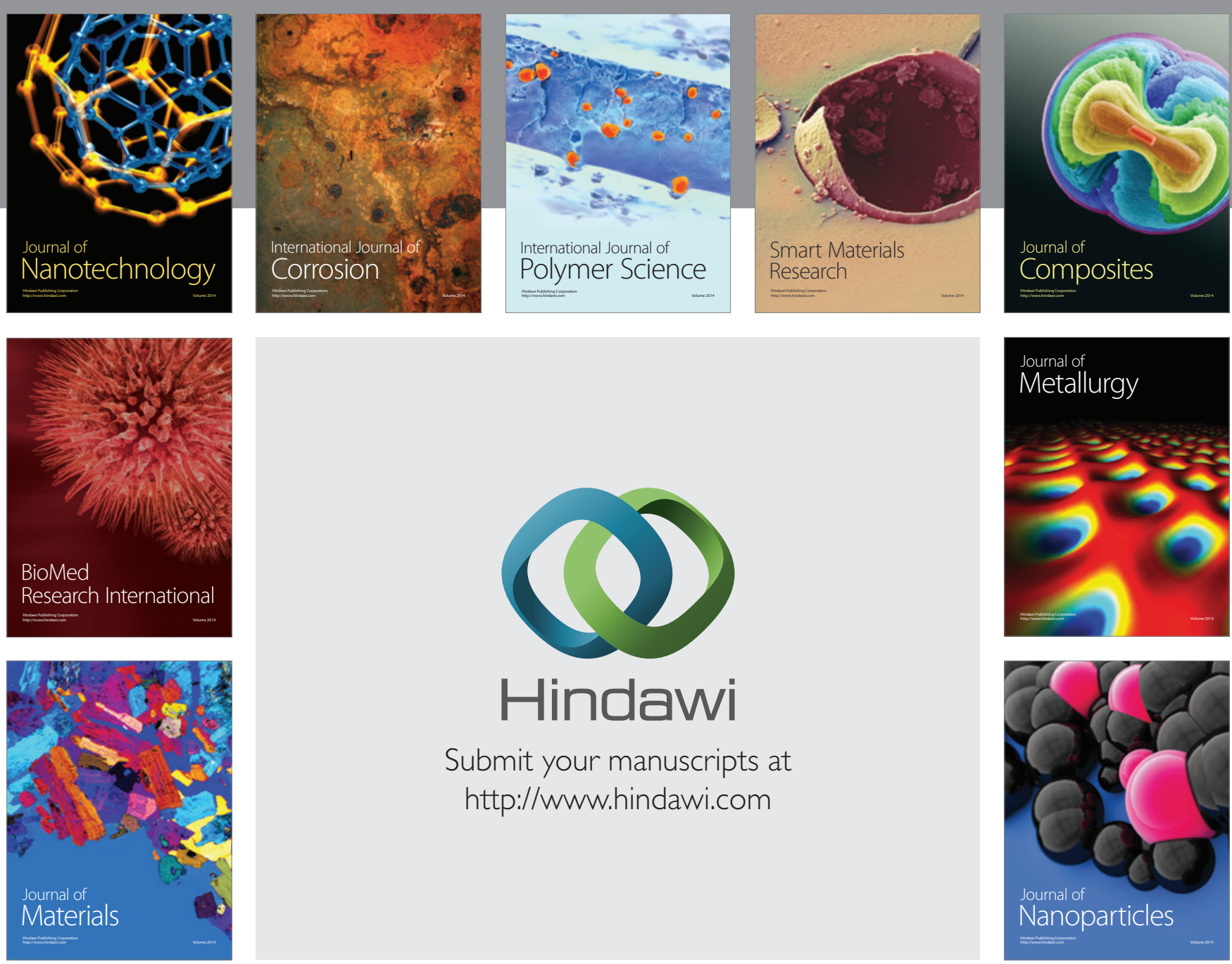

Submit your manuscripts at http://www.hindawi.com
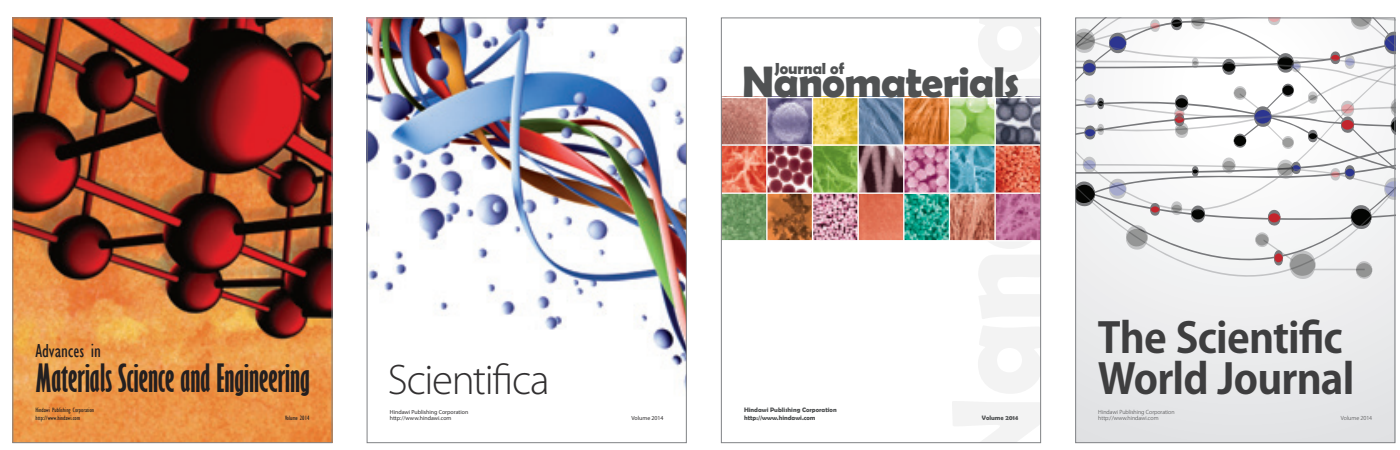

\section{The Scientific World Journal}
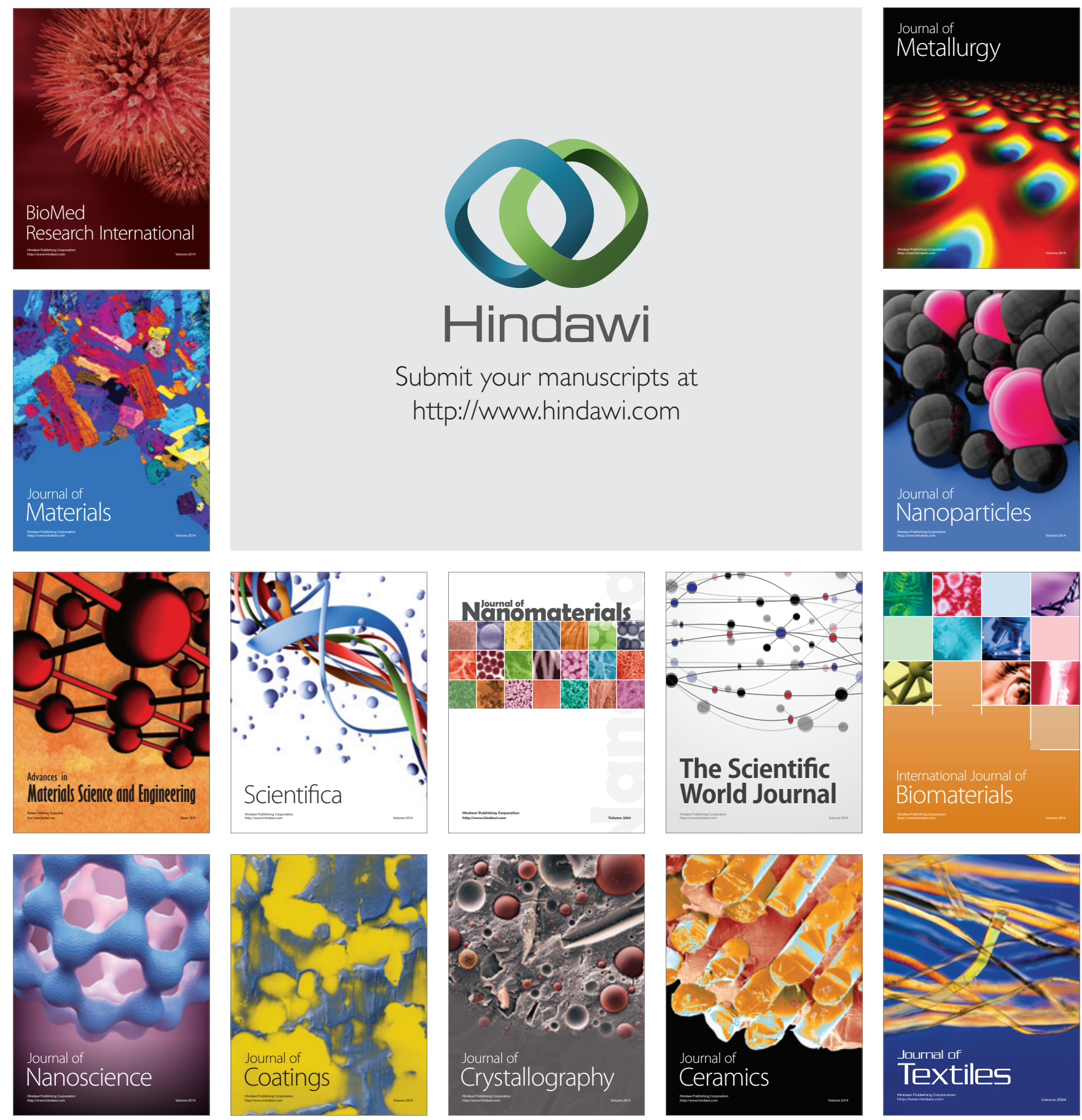